\title{
MESILATO DE IMATINIB INDUZINDO DERRAME PLEURAL BILATERAL E TAMPONAMENTO CARDÍACO
}

\author{
IMATINIB MESYLATE INDUCING BILATERAL PLEURAL EFFUSION AND \\ PERICARDIC EFFUSION
}

DOI: $10.5380 /$ rmu.v2i1.40674

Rodrigo Melo Kulchetscki ${ }^{1}$, Aline Cristini Vieira ${ }^{1}$, Victor Borsani S. Cury ${ }^{1}$, Juliane Rompkoski ${ }^{1}$, Felipe D. Dos Santos ${ }^{1}$, Gustavo Lenci Marques ${ }^{1}$, Francisco Luiz Gomide Mafra Magalhães ${ }^{1}$

\section{RESUMO}

Introdução: A leucemia mieloide crônica (LMC) é uma patologia marcada pela síntese de uma tirosina quinase BCR-ABL constitucionalmente ativa. Com o surgimento dos inibidores da tirosina quinase houve uma grande melhora no prognóstico dos pacientes com o tratamento da LMC. A ocorrência de derrame pleural e pericárdico é mais descrita com o uso do dasatinibe, sendo rara com o uso do imatinibe e nilotinibe. Devido à raridade do caso, iremos apresentar um relato de uma paciente de 19 anos em uso de imatinib, a qual apresentou efusões pleural e pericárdica relacionadas à terapia. Relato: Paciente do sexo feminino, 19 anos, apresenta-se ao Pronto Atendimento do Hospital de Clínicas da UFPR em julho de 2012 com queixa de dispneia e dor torácica ventilatório-dependente, com piora nos últimos cinco dias. Ao exame, observou-se murmúrio vesicular diminuído bilateralmente em bases, bulhas cardíacas hipofonéticas, abdome ascítico e edema de membros inferiores abaixo dos joelhos. Foram solicitados exames complementares que demonstraram derrame pericárdico importante associado a tamponamento cardíaco, necessitando de janela pericárdica. Seis dias após o ocorrido a paciente foi reavaliada e internada, sendo submetida à drenagem torácica fechada, com drenagem de $2450 \mathrm{ml}$ no total de 9 dias de internamento. A paciente tinha o diagnostico de LMC t(9,22) desde 2008, tendo sido iniciado o tratamento com Gleevec ${ }^{\circledR} 400 \mathrm{mg} / \mathrm{dia}$ em março de 2009, tendo sido acompanhada e tendo apresentado apenas efeitos colaterais aceitáveis; a dose da medicação fora mantida até novembro de 2011 quando se optou por aumentar a dose para 600 $\mathrm{mg} /$ dia graças a uma resposta molecular subótima. Após a resolução e investigação do quadro, outras causas foram excluídas e optou-se pela introdução do nilotinibe para controle da doença. Conclusão: Este relato ressalta a relevância dos efeitos adversos relacionados ao uso do imatinib no tratamento da LMC.

Palavras-chave: fibrose cística, tuberculose, Chopin.

\section{ABSTRACT}

Chronic myeloid leukaemia (CML) is a disease defined by the synthesis of the tyrosine kinase BCR-ABL1 constitutionally active. With the development of a tyrosine kinase inhibitor there was a great improvement in the prognosis of patients in treatment of CML. The development of pleural and pericardial effusion is most common with dasatinib and more rare with imatinib and nilotinib. Because of rarity of the case we will report a 19 years old pacient that presented pleural and pericardial effusion due to the use of imatinib mesylate. Report: Female pacient, 19 years old, goes to the Emergency Department of UFPR Clinical Hospital in july of 2012 with dyspnea and ventilatory dependent pain that got worse in the past five days. On examination, there was diminished breath sounds bilaterally bases, hypophonetic heart sounds, abdominal ascites and lower extremity edema below the knees. Additional tests were requested which showed significant pericardial effusion associated with cardiac tamponade requiring pericardial window. Six days after the incident, the patient was re-evaluated and admitted, submitted to closed drainage, drainage with $2450 \mathrm{ml}$ total of 9 days of hospitalization. The patient had a diagnosis of CML t (9.22) since 2008, having started treatment with Gleevec $^{\circledR} 400 \mathrm{mg} /$ day in March 2009, having been monitored and having only made acceptable side effects; medication dose had been kept until November 2011, when it was decided to increase the dose to $600 \mathrm{mg} /$ day thanks to a suboptimal molecular response. After the resolution and investigation of the framework, other causes were excluded and opted for the introduction of nilotinib for disease control. Conclusion: This report highlights the relevance of adverse effects related to the use of imatinib in CML treatment.

Key words: cystic fibrosis, tuberculosis, Chopin.

1-Universidade Federal do Paraná

Contato do Autor / Mail to:

Rodrigo Melo Kulchetscki - rodrigo_mk1@yahoo.com.br

Rua General Carneiro, 181 - Alto da Glória Curitiba - PR, 80060-900, 10 Andar. 


\section{INTRODUÇÃO}

A leucemia mieloide crônica (LMC) é uma patologia marcada síntese de uma tirosina quinase BCR-ABL constitucionalmente ativa. resultante de uma translocação balanceada entre os cromossomos 9 e 22, chamado de cromossomo Philadélphia ${ }^{1}$. Com 0 surgimento dos inibidores da tirosina quinase houve uma grande melhora no prognóstico dos pacientes com o tratamento da LMC.

O imatinibe foi introduzido como primeira linha de tratamento há cerca de 10 anos, e apesar de sua eficácia, uma parcela dos pacientes desenvolve resistência ou apresenta efeitos adversos ao seu uso. A ocorrência de derrame pleural e pericárdico é mais descrita com o uso do dasatinibe, sendo rara com o uso do imatinibe e nilotinibe.

Devido à raridade do caso, iremos apresentar um relato de uma paciente de 19 anos em uso de imatinib, a qual apresentou efusões pleural e pericárdica relacionadas à terapia.

\section{RELATO DO CASO}

H.P.B, sexo feminino, 16 anos, branca, apresentou-se ao ambulatório de hematologia do Hospital de Clínicas da Universidade Federal do Paraná em dezembro de 2008 por queixa de dor no peito.

A paciente tinha sido recém diagnosticada com Leucemia Mielóide Crônica (LMC) t $(9,22)$, tendo aberto o quadro cerca de 40 dias antes, com dor torácica importante, leucocitose 300.000 e esplenomegalia com baço a $17 \mathrm{~cm}$ do rebordo costal esquerdo. Na época em tratamento com Hidroxiuréia 1 comprimido VO por dia. Nega outros antecedentes médicos ou cirúrgicos. Nega tabagismo, alcoolismo e uso de drogras ilícitas.

Ao exame, apresentou-se em bom estado geral, corada, hidratada, eupneica. O exame abdominal mostrou um abdome flácido, indolor à palpação. 0 baço foi palpável a $3 \mathrm{~cm}$ do rebordo costal esquerdo. 0 restante do exame segmentar não mostrou alterações.

Os exames laboratorias da admissão mostravam-se normais, com 5820 leucócitos, exceto por plaquetopenia (68.000) e anemia (hemoglobina 10,3; reticulócitos 3,2). A medicação foi mantida e a paciente foi orientada a acompanhar no ambulatório. Foi-se instituído o tratamento com Gleevec ${ }^{\circledR} 400$ $\mathrm{mg} /$ dia em março de 2009. A paciente foi acompanhada e observaram-se apenas efeitos colaterais aceitáveis. Em novembro de 2011, apresentava resposta citogenética completa porém resposta molecular sub-ótima, sendo optado por aumentar a dose do Gleevec ${ }^{\circledR}$ para $600 \mathrm{mg} /$ dia.

Em 04 julho de 2012, a paciente apresentouse ao PA do Hospital de clínicas com queixa de dispneia e dor torácica ventilatório dependente, com piora nos últimos cinco dias, associado a tosse seca.

Ao exame, observou-se murmúrio vesicular diminuído bilateralmente em bases, bulhas cardíacas hipofonéticas, abdome ascítico e edema de membros inferiores abaixo dos joelhos.

Foram solicitados um ecocardiograma, uma ecografia de abdome, um raio $x$ de tórax $e$ proteinúria de 24 horas, que demonstraram derrame pericárdico importante associado a tamponamento cardíaco. A paciente foi, então, submetida a janela pericárdica.

Seis dias após o ocorrido a paciente foi novamente admitida no PA para reavaliação, sendo, então, internada e submetida a toracocentese com aspiração de $600 \mathrm{ml}$ e, em seguida $500 \mathrm{ml}$, de líquido serossanguinolento, sendo internada e submetida a drenagem torácica fechada, com drenagem de $2450 \mathrm{ml}$ no total de 9 dias de internamento.

Recebeu alta com Predinisona $60 \mathrm{mg} / \mathrm{dia}$, Keflin e sem Gleevec ${ }^{\circledR}$. No acompanhamento, solicitouse FAN e anti-DPN, ambos negativos.

No acompanhamento, observou-se leucocitose de 15.520 e optou-se pela introdução do nilotinibe para controle da doença.

No dia 24/09/12, relata nova dor retroesternal, sendo solicitados ecocardiograma, raio $x$ de tórax, todos normais. Optou-se por acompanhamento ambulatorial e reavaliação em 30 dias.

\section{DISCUSSÃO}

Com o surgimento dos inibidores da tirosina quinase houve uma grande melhora no prognóstico dos pacientes com o tratamento da LMC. O imatinib foi introduzido como primeira linha de tratamento há cerca de 10 anos, e apesar de sua eficácia, uma parcela dos pacientes desenvolve resistência ou apresenta efeitos adversos ao seu uso ${ }^{2}$.

Devido a esse fato, novos inibidores tirosina quinase foram desevolvidos, tais como dasatinib, nilotinib e bosutinib ${ }^{3}$. O uso do dasatinib (100 mg, uma vez ao dia) e nilotinib ( $400 \mathrm{mg}, 2$ vezes ao dia) foram aprovados nos Estados Unidos e Europa como terapia em pacientes resistentes ou intolerantes ao imatinib (dasatinib para todas as fases da LMC e nilotinib para fase crônica e fase acelerada). Nos Estados Unidos, dasatinib (100mg, uma vez ao dia) e nilotinib (300mg, 2 vezes ao dia) foi aprovado para pacientes recém diagnosticados com a fase crônica da LMC².

Geralmente, esses medicamentos são bem tolerados, e segundo o estudo IRIS, os efeitos adversos tendem a ocorrer precocemente ${ }^{4}$.

Sabe-se que é raro a ocorrência de efusão pleural durante o uso de imatinib e nilotinib, e mais 
comum no uso de dasatinib ${ }^{5,6}$. Isso foi comprovado no estudo DASISION, uma vez que o imatinib apresentou mais retenção de fluído, edema superficial, mialgia, vômitos e rash que o dasatinibe, enquanto este apresentou mais efusões pleurais (17\%, contra $0 \%$ do imatinib) ${ }^{7}$.

Além disso, em relação a cardiotoxicidade, em 2006 foi publicado um relato de dez pessoas que desenvolveram insuficiência cardíaca congestiva durante o uso de imatinib, que segundo o autores seriam em decorrência da inibição da atividade da $A B L$ no tecido cardíaco ${ }^{8}$.

\section{CONCLUSÃO}

Como o imatinib pode ter efeito nos fluídos hemodinâmicos e um potencial de retenção em espaços viscerais, sugerimos que seja realizado um monitoramento atento dos pacientes tratados com imatinib em altas doses.

\section{REFERÊNCIAS}

1. Colleoni GWB., Satake M, Borovik CL, Kerbauy J, Yamamoto M. Leucemia mielóide aguda Ph1-positivo de novo ou crise blástica de leucemia mielóide crônica? Análise molecular e evolução clínica de um caso. Rev. Assoc. Med. Bras. 1998; 44(3):253-255. http://dx.doi.org/10.1590/S0104-42301998000300015

2. Wei G, Shamudheen R, Liu D. First-line treatment for chronic myeloid leukemia: dasatinib, nilotinib, or imatinib. J Hematol Oncol, 2010 3:47. doi:10.1186/1756-8722-3-47

3. Saglio G, Baccarani M. First-line therapy for chronic myeloid leukemia: new horizons and an update. Clin Lymphoma Myeloma Leuk. 2010;10:169-176.

doi: 10.3816/CLML.2010.n.026. [PubMed]
4. Kantarjian HM, Larson RA, Guilhot F, O'Brien SG, Druker BJ. on Behalf of IRIS Study Group. Declining rates of adverse events (AEs), rare occurrence of serious AEs, and no unexpected long-term side effects at 5 years in patients with newly diagnosed chronic myeloid leukemia in chronic phase initially treated with imatinib in the international randomized study of interferon vS STI571 (IRIS) Blood. 2006;108(Suppl) abstract 2136.

5. Masiello D, Gorospe G, Yang AS. The occurrence and management of fluid retention associated with TKI therapy in CML, with a focus on dasatinib. J Hematol Oncol. 2009;2:46. doi: 10.1186/1756-8722-2-46.

6. Wong SF. New dosing schedules of dasatinib for CML and adverse event management. J Hematol Oncol. 2009;2:10. doi: 10.1186/1756-8722-2-10.

7. Kantarjian $\mathrm{H}$, Shah NP, Hochhaus A, Cortes J, Shah S, Ayala M, Moiraghi B, Shen Z, Mayer J, Pasquini R, Nakamae $\mathrm{H}$, Huguet $\mathrm{F}$, Boque C, Chuah C, Bleickardt E, Bradley-Garelik MB, Zhu C, Szatrowski T, Shapiro D, Baccarani M. Dasatinib versus imatinib in newly diagnosed chronic-phase chronic myeloid leukemia. N Engl J Med. 2010; 362:2260-2270. doi: 10.1056/NEJMoa1002315.

8. Kerkela R, Grazette L, Yacobi R, Iliescu C, Patten R, Beahm C, Walters B, Shevtsov S, Pesant S, Clubb FJ, Rosenzweig A, Salomon RN, Van Etten RA, Alroy J, Durand JB, Force T. Cardiotoxicity of the cancer therapeutic agent imatinib mesylate. Nat Med. 2006;12:908-916. doi: $10.1038 / \mathrm{nm} 1446$. 